\title{
Modelling and Simulation, Mobile Solutions and Cloud Computing: A Solution for Intelligent Management of a Small and Medium-Sized Baking Business
}

\author{
Hugo de Souza Ribeiro ${ }^{\text {, Braulio Roberto Gomes Marinho Couto }}{ }^{2}$, Diva Souza e Silva \\ Rodrigues $^{3}$, Luiz Melk de Carvalho ${ }^{4}$, Flávio Henrique Batista de Souza ${ }^{5 *}$
}

Centro Universitário de Belo Horizonte - UNIBH, Brazil

flabasouza@yahoo.com.br

\begin{abstract}
The sale of products during the pandemic by COVID-19 was severely affected. Several small businesses and industries have reduced their productivity or even closed down. As a result, companies that still survive this complicated period must rely on intelligent (but accessible) resources to guide their survival on the market. The purpose of this paper is to demonstrate an integrated and affordable tool, based on technologies such as mobile applications, Cloud Computing and discrete event simulation to guide small manufacturers in the bakery sector. The solution has a robust mobile application to control the inventory, sales and product prices (with customer records, prices and reports control). Its database is managed via cloud computing. However, the great contribution is the connection of this solution with a simulator based on Stochastic Timed Petri Nets, which is integrated with the mobile application through the cloud commuting. This tool manages to model and simulate each type of product, with its time and resources spent during the manufacturing process, with production hierarchy according to the production priority that the manufacturer requests. Its focus is the intelligent management and identification of bottlenecks and evaluation of production scenarios. In a practical application in a small real industry, in the middle of a pandemic period, it was possible to make optimizations that still keep the enterprise open, such as evaluations of equipment purchases, reassessment of processes (which yielded a 9\% increase in productivity without any financial investment and just maintenance of some work positions) and readjustment of material purchases.
\end{abstract}

Keywords: Stochastic timed petri nets, cloud computing, mobile applications, baking business, intelligent management. 


\section{$3^{\text {rd }}$ International Conference on Research in SCIENCE, ENGINEERING AND TECHNOLOGY}

11-13 March, 2021

Oxford - United Kingdom

\section{Introduction}

The advances in technology has been exceptionally fast in this century. Information Technologies have become more powerful and more accessible contributing heavily to the increase in overall investment and efficiency of labour and capital. Business Intelligence and Analytics have become a fundamental reference of many organizations to take informed decisions. Despite the importance of small and medium-sized enterprises to all world economies, research studies focus mostly on large enterprises (UNCTAD, 2016).

To contribute to closing this gap, this study provides a comprehensive review of the currently situation of mobile solutions. Despite much interest in Business Intelligence and Analytics, shows that small and medium-sized enterprises are still lagging in the proliferation of business management and control software. Small and medium-sized enterprises (SMEs) play major economic and social roles because they account for about ninety percent of businesses and more than fifty percent of employment worldwide according to the International Finance Corporation (IFC, 2015, p. 34)

Discrete Event Simulation (DES) is an extremely versatile technique and a method of simulating the behaviour and performance of a real-life process, facility or system and due to these aspects it allows companies to test as many scenarios as necessary in order to find the ideal model for application (Cassandras and Lafortune, 2009). The Petri net is a graphical and mathematical modelling tool applicable to several systems. It is a well-established formal specification technique, widely used, being suitable for modelling systems that have parallel, concurrent and non-deterministic activities allowing to evaluate the structure and dynamic behaviour of the modelled system (Reis et al., 2018; Lisboa et al, 2019).

The main achievement of this research is to make possible, through the consolidation of technology data such as cloud computing, mobile solutions and modelling and simulation to develop a solution for the intelligent management of a small and medium-sized bakery enterprise, using android based smartphone technology. The purpose of developing this mobile application is to manage a company of sprinkle biscuit, originated from cassava starch, including management, purchases, production, sales, logistics and finance. Currently, everything is accomplished using spreadsheets, papers, notes and it does not have an automated and reliable control for any of the production, entry and exit stages of the company (a recurring administration method in the Brazilian industry).

The starch biscuit is traditional in practically every Brazilian region and with the help of small machines it became possible to start a mini factory with low initial investment, which has aroused the interest of new entrepreneurs. Basically, the production process is simple: It all starts with the production of the biscuits, then they are taken to the oven, packaged and supplied on the market. Reliable prediction of sales is of immense benefit to a business, because it can improve the quality of the business strategy and decrease costs due to waste, thereby increasing profit. To improve the competitiveness of the enterprise, the manager must take correct decisions using the available information. This "forecasting" is viewed as an important part of 


\section{$3^{\text {rd }}$ International Conference on Research in SCIENCE, ENGINEERING AND TECHNOLOGY}

11-13 March, 2021

Oxford - United Kingdom

decision making. With the advent of COVID-19, decision making can define the success or failure of the business.

\section{Methods}

The implementation methodology, in this experimental research, consisted of three steps. The first step was performed based in assimilate the productive process to propose a development of a mobile tool for inventory management data. Then an integration process with the cloud was carried out, where a cloud database consolidates the data. Finally, a modelling and simulation was performed using Petri nets.

An up to date appraisal was accomplished through a technical visit to the company that operates in the bakery market of the city of São João do Manhuaçu (Minas Gerais/Brazil). A data collection process was carried out in the company, which executed for four months, in loco, by the authors, through documentary data extracted from the company processes. The time of production of starch biscuit was analysed, in order to have a greater precision of the collected data. The monitoring process was performed in all stages of this process, from raw material order, stages, time to arrival, manufacturing, quality link, validity, packaging, labelling, to delivery to the customers. This study sought to design, develop and test an information system to meet information needs of starch biscuit manufacturing. Therefore, a mobile solution was developed to act on the stock management process to optimize the information gathering.

Thus, a simulation structure was implemented, using the Petri Nets tool, which can not only abstract reality, but also propose scenarios, in order to evaluate the effects of buying and selling in inventory. It was analysed which types of flavours are idle in inventory, which are the ones with the highest turnover, the periods of stock of biscuits and which lead times for delivery after purchase order. The company provided data on the products it works with, in quantities and purchase and sale prices, over the four-month period from January to April of 2020. Through the data provided it was achievable to perform an analysis of how products enter and leave the stock. To conclude, the review of the present article is to make possible a production process through the Petri Net, with data provided by the company called Delicate ${ }^{\circledR}$ and with a flow diagram characterizing the complete process, from the raw material to the final consumer.

\section{Results}

\subsection{Optimization Demand Analysis}

For the process of data collection and storage, an application was developed using the Android studio platform, which through simple interfaces allows the user to record the incoming and outgoing production inputs and products from the stock, providing control, checking and increasing the reliability of information. The sales structure that works during early period of a business is different from what works when the business is growing, during its maturity. In addition, the application provides relevant users with clarifications regarding the total stock value and movement history. Sales representative responsibilities include selling products using solid arguments to prospective customers, performing cost-benefit analyses of 


\section{$3^{\text {rd }}$ International Conference on Research in SCIENCE, ENGINEERING AND TECHNOLOGY}

existing and potential customers and maintaining positive business relationships to ensure future sales.

During the period from January to May of 2020, the company analysed the data before and during the pandemic. The data was collected from the traditional analysis, invoices and receipts. Unfortunately, mismanagement and incorrect storage of invoices and contacts can cause big losses to small/medium companies and one of the aggravating factors is to restructure all companies affected by the Covid-19 to think before investing on the platform that will improve the management of the business effectively. The objective was to evaluate, not only how the old process did not have the effective control of unpaid invoices, but to evaluate an automated report for information control.

The relevance of this study have a great benefit for the information in the company, since the factory did not have a report of control of invoices without payments, a report of sales by companies, it was not able to control the biscuits production and a broader administration, as well as accessible tools for inventory control. The demand which was raised migrates to a system with capacity to register customers, inputs, products, inventory control, sales and reports with much information for an improvement management and productivity of the company.

\subsubsection{Manufacturing Process Flow Evaluation}

To provide a detailed diagnosis, the manufacture of the main products from Delicate ${ }^{\circledR}$ were monitored and analysed continuously to sell to the market: Traditional, Onion and Cheese flavours. However, the process has some production bottlenecks that can be easily identified and solved with a strong strategic planning. It is very important to understand that each individual product requires specific treatment according to their peculiarities.

The main obstacle found in the Delicate ${ }^{\circledR}$ biscuit manufacturing process is the continued use of employees' manual work, in other words, the machinery shortage, as shown in figure 1 . The manufacturing process of Delicate ${ }^{\circledR}$ is a feasible solution for a small business. This has been accompanied by the entire production process of Traditional flavour biscuit as well as implementing the necessary controls to guarantee the quality of the final product. First, the raw material is weighed in around 15 minutes and mixed in a planetary mixer (Figure 1 - a), all this process takes approximately 30 minutes. After the admixture, the process starts. The biscuit dough is placed in a manual multidrop depositor (a manual handling machine to presses the dough and shapes the biscuits into the baking tray - Figure 1-b), with a maximum capacity of $15 \mathrm{Kg}$ and running time of approximately 5 minutes, the biscuits are dripped onto the tray and are taken to the oven to bake. 


\section{$3^{\text {rd }}$ International Conference on Research in SCIENCE, ENGINEERING AND TECHNOLOGY}

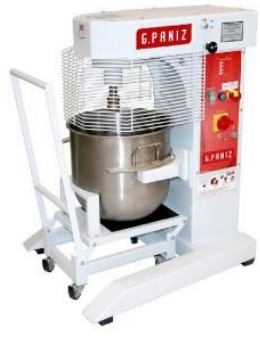

(a) Mixer

Source: Authors, 2020.



(b) Manual Handling Machine

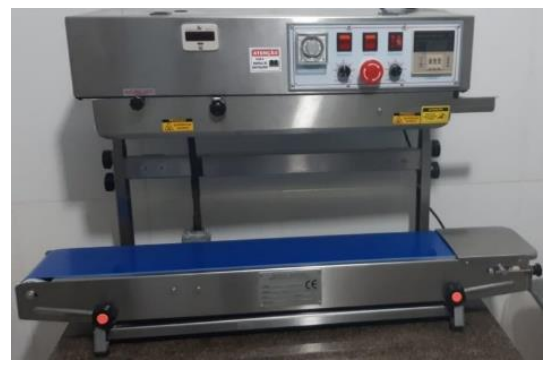

(c) Sealer Machine

The machine operation is simple, just put the dough in a Brushed Stainless-Steel Bowl and lower an activation lever to produce the biscuits modelled. In 5 minutes, 8 kilos of biscuits are ready for baking. After this period of the previous process, the baking trays are transferred to the industrial oven through a rack oven, it can hold up to 56 baking trays (40"X60") of product. The time of this process is approximately 1 minute. The oven has the capacity to bake 8 kilos of dough in 26 minutes at a temperature of $150^{\circ} \mathrm{C}$. Then, the rack oven is removed and left to cool for approximately 5 minutes and all baked biscuits are removed from the baking trays.

In this step, the weighing of the biscuits begins and they are placed in individual package, defined by size, (in this example, 200 grams). This part takes an average time ranging between 5 to 10 seconds per package, so the process can go through the next stage, the sealing system. The thermal sealing process is carried out by a sealer machine, Figure 1-c, whose basic principle is the fusion of the plastic film to the fibres of the porous material. This fusion is accomplished by "melting" one of the layers of the film, which after being pressed against the porous material, for a certain time, penetrates between the fibres and causes the anchoring. Each package takes an average of 2 to 5 seconds to seal. In the next step, it is the process of individual nutritional labelling of the packages, which also takes an average of 2 to 5 seconds to label each unit. Properly labelled with all the information, production batch, expiry date etc., finally, the last stage of the process starts and the individual packages are placed in bales with 15 units each, in 200 grams size. This part takes, on average, between 1 minute and 30 seconds and 2 minutes. Then, the dispatch is made to the sales representatives of the company, using the routes according to the location/region of the sellers.

\subsection{Mobile Solution}

After the evaluation of the manufacturing and sales process, the mobile solution for inventory and sales control was implemented. Figure 2 shows the diagram of the mobile solution architecture. The interfaces can be seen in tables 1 and 2, where screenshots of each graphical interface are available and Figure 3 demonstrates the utilization flowchart. The app has as an initial screen with an interface that allows the user to login to the app through their registration within the company and a password previously registered. If the user does not have a registration, it is necessary request to the administrator, which is the only profile that is responsible to create a new user (for security reasons). 
Figure 2: Mobile Application Structure Diagram

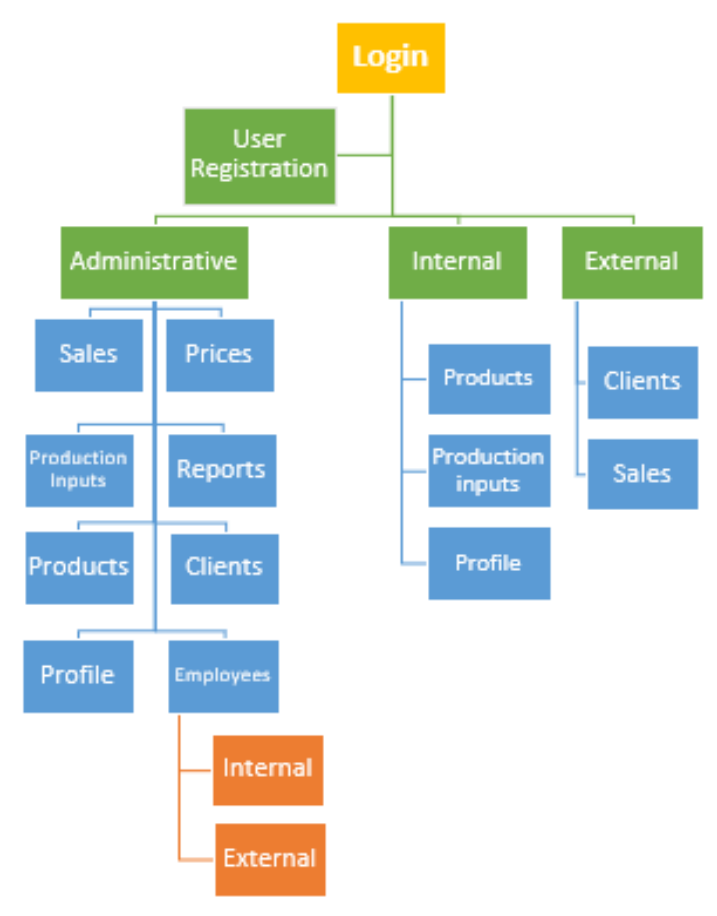

Source: Authors, 2020

The user must log in using their registration number with the company upon admission, as well as their full name, social security number (CPF), email and select one of the options related to the work sector: Internal or external profiles will allow what type of access and what features the user will have available. All entries will be directed to key users by email for verification to be able to $\log$ in. Upon log in, the user is directed to the menu page, according to their registered profile. The internal profile menu will be used by internal employees with permission to use the Delicate ${ }^{\circledR}$ App. They will have access only to work-related functionalities, intended for production inputs, products and inventory control, which are:

Administration profile: the administrative profile menu will have access to all features of the application, which are:

- Clients (Input and Inquiry): The required information must be register as name, CNPJ (identification number issued to Brazilian companies by the Department of Federal), contact number and e-mail. Other information is required but not essentials. The registration of clients must be done before sales.

- Products: where the registration of production is performed with the input and output of each flavour produced. 


\section{$3^{\text {rd }}$ International Conference on Research in SCIENCE, ENGINEERING AND TECHNOLOGY}

\section{Mociset}

11-13 March, 2021

Oxford - United Kingdom

Figure 3: Mobile Application Purchase Process flow

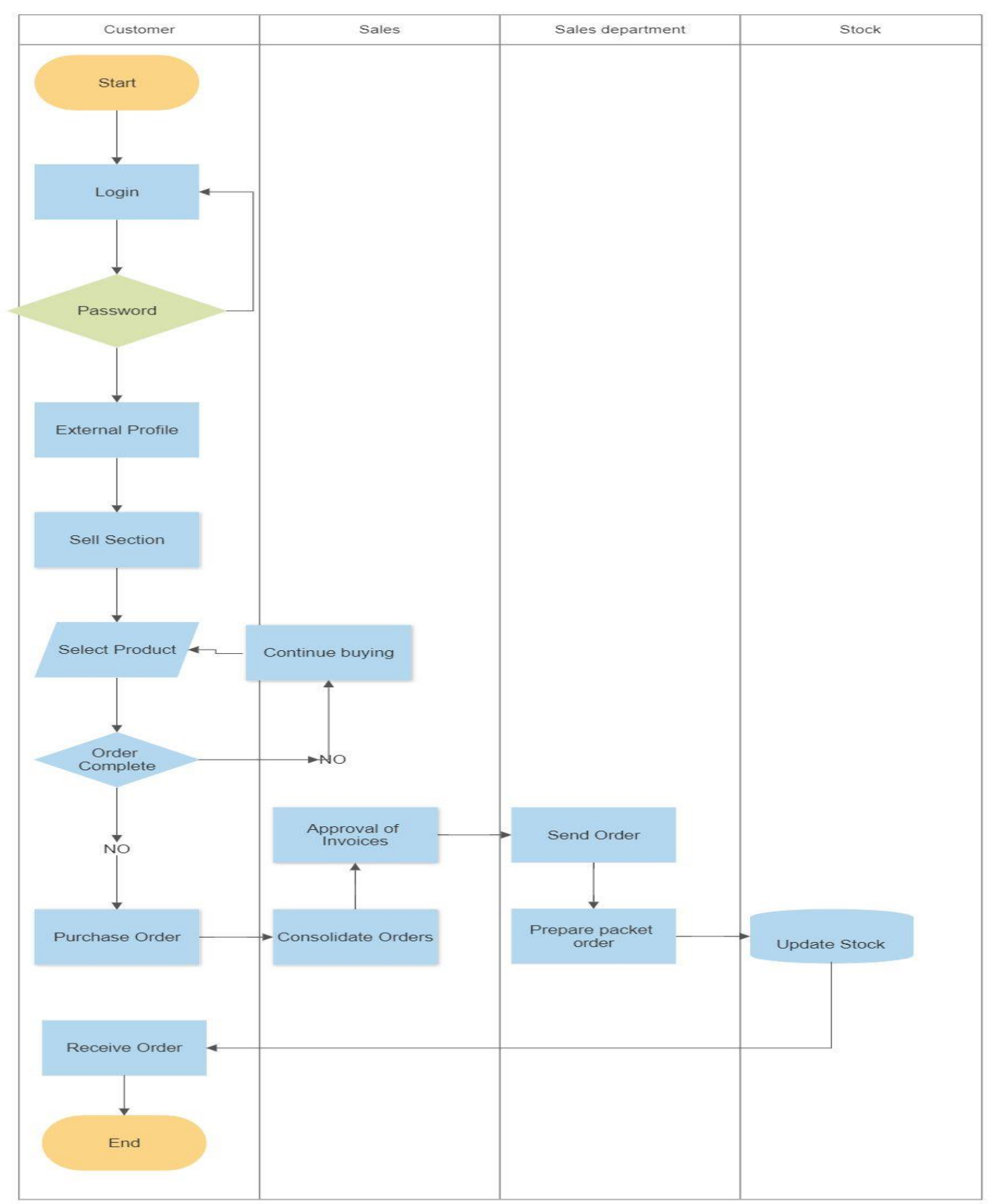

Source: Authors, 2020.

- Production Inputs (Inventory Input and Inquiry): The purpose of this option is to search for the availability of an item already registered in stock through a floating menu, reducing the need to access the physical inventory unnecessarily.

- Prices: Price list of each products and flavours. The only profile that can register the prices is the administrator profile. Each external profile has a specific price list. The region can affect the price due to different reasons as delivery or specificity. 


\section{$3^{\text {rd }}$ International Conference on Research in SCIENCE, ENGINEERING AND TECHNOLOGY}

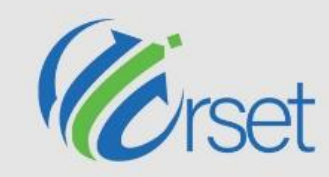

11-13 March, 2021

\section{Oxford - United Kingdom}

- Sales: This function allows the administrator to check all the sales and generating a control and history. All the sales will be registered in a database for a study to show which clients or regions can be improved for future sales.

Table 1 -Interfaces Screenshots (Part 1)

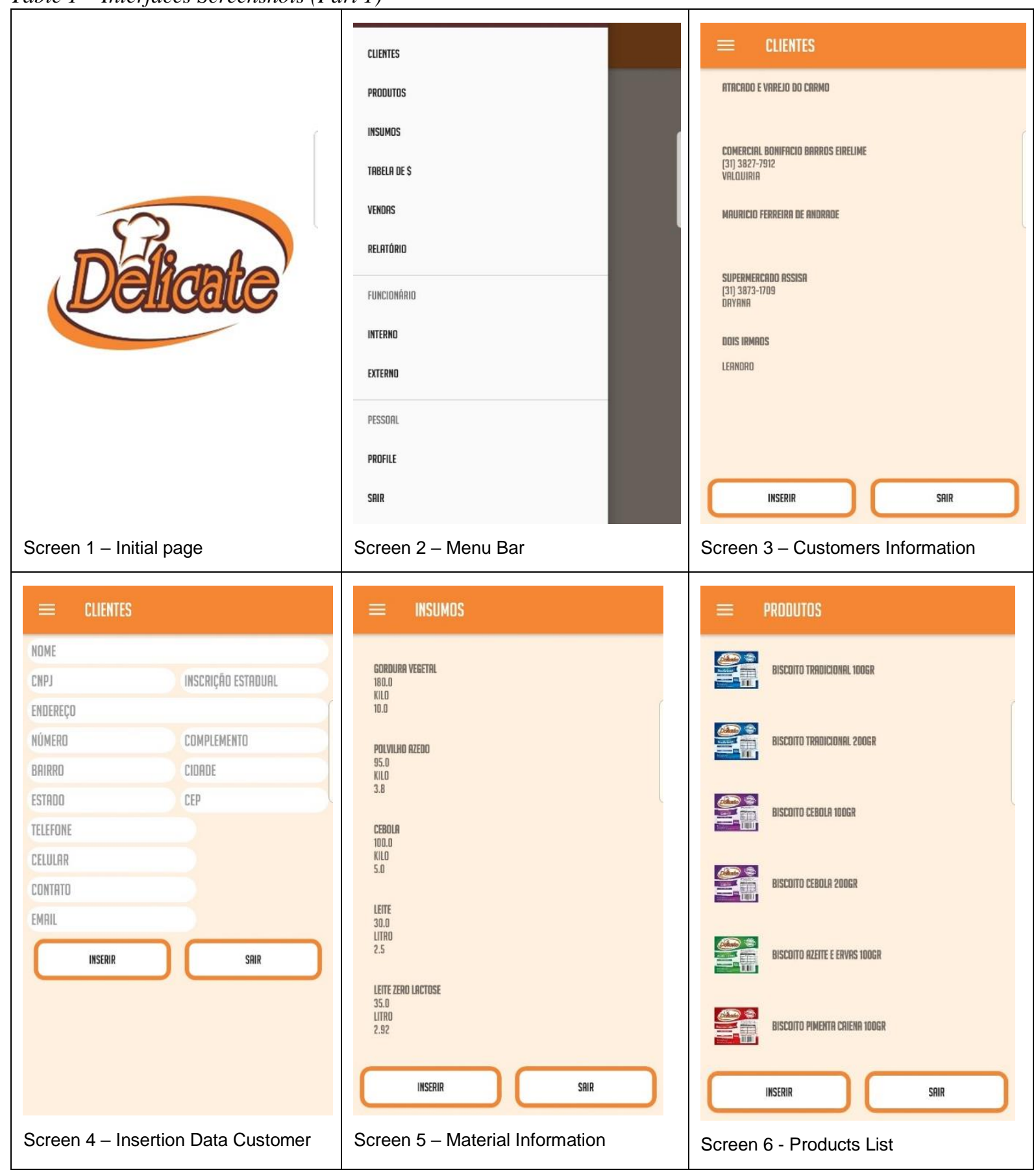

Source: Authors, 2020.

- Reports (Movement History): This functionality allows the administrator user to see all reports in any period, generating a control and history for all information. 


\section{$3^{\text {rd }}$ International Conference on Research in SCIENCE, ENGINEERING AND TECHNOLOGY}

\section{Viciost}

11-13 March, 2021

Oxford - United Kingdom

- Employees: Control list of internal and external profiles of the employees.

Internal profile: the internal profile menu will have access to products features of the application, which are:

Table 2 - Interfaces Screenshots (Part 2)

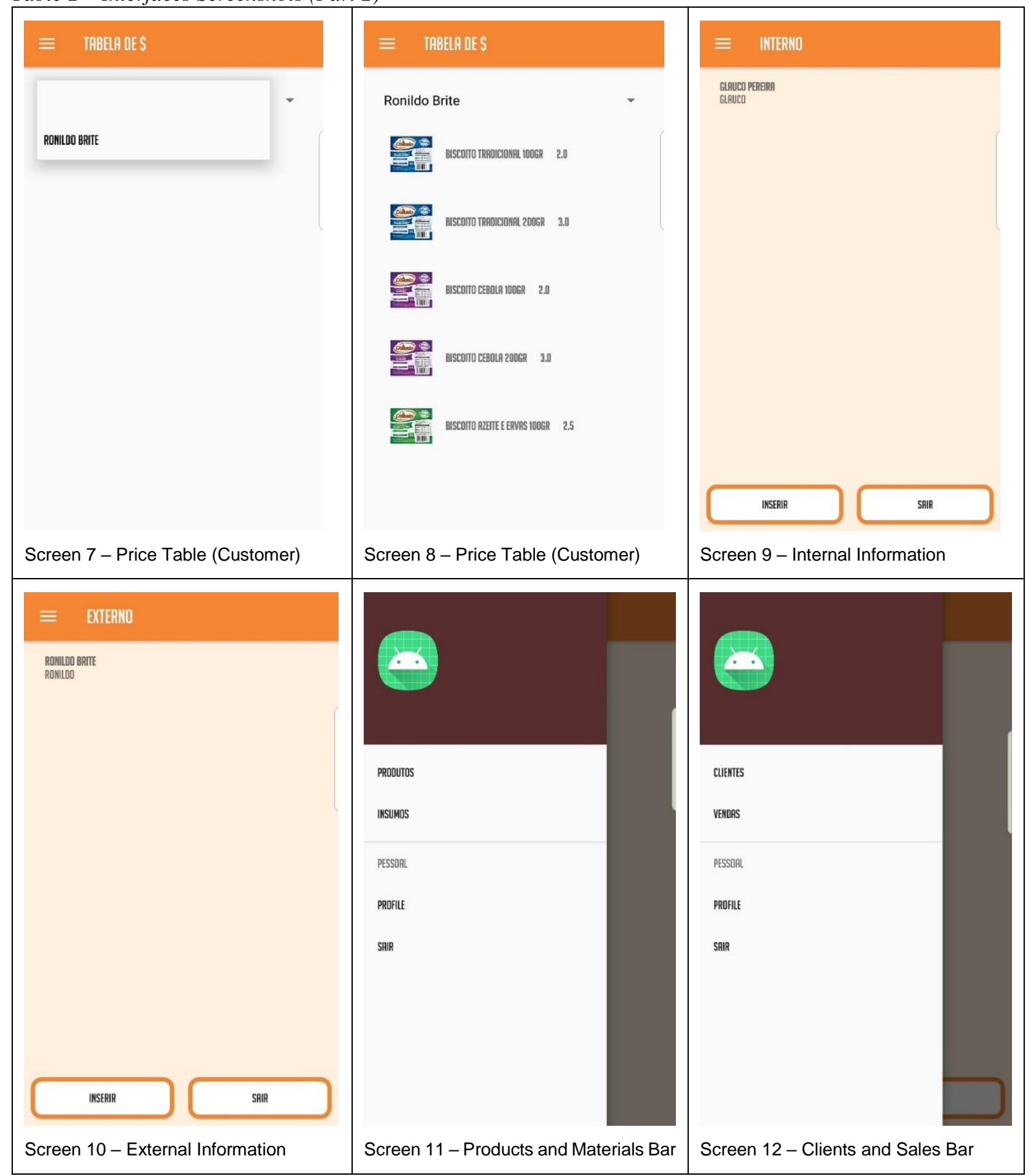

Source: Authors, 2020 


\section{$3^{\text {rd }}$ International Conference on Research in SCIENCE, ENGINEERING AND TECHNOLOGY}

11-13 March, 2021

Oxford - United Kingdom

- Products

- $\quad$ Production Inputs (Inventory Input and Inquiry)

- Reports (Movement History): This functionality allows the user to see inventory movements in each period, generating a control and history of turnover of products or production inputs that may influence some strategic decisions, such as purchase of production input or even outputs of products sales to specific regions.

External profile: the external profile menu will allow access to:

- Clients (Input and Inquiry)

- Sales: This function allows the user to register the sales for a customer, including the kinds of products and quantity for each flavour and generating a control and history. All the sales will be registered in a database for a study about what clients or regions can be improved for future sales. The salesman must choose the customer first and make the list of products as requested by the customer. If the amount in the stock is less than the amount chose by the clients, the sales cannot be registered, and the app shows a message warning this situation.

- Reports (Movement History): This functionality allows the user to see all sales movements in each period, generating a control and history of turnover of sales per clients, sales dates or places that may influence some strategic decisions, such as the amount of each kind of flavours should be designated for sales to specific regions.

The purchase process is carried out through the purchase order which is sent online to the administration and sales department. This request is prepared by the internal team, based on the stock analysis, where the quantity of each products and flavours are verified. Usually the request is made in real time. The online selling is crucial, the seller/External profile being on the site, using the sell section to manage orders, update items, and respond to the customers on the go. To execute this project, a relational database was used on the MySQL server, for data storage and management with SQlite, that implements a self-contained, serverless, zero-configuration, transactional SQL database engine.

\subsection{Modelling and Simulation Analysis}

The mobile solution is connected via cloud computing to a Petri network-based simulation model. The consolidation of sales time data, resource quantities and process times are stored in the cloud, and then guide the simulation models. The Petri nets were used to simulate demands from customers or regions, to answer questions such as: is there any input missing? what is missing? When? Consequently, more advantageous contract strategies can be developed for the factory. The transition that was considered non-timed is the time for baking the Delicate $\mathbb{R}$ Biscuits, which is automatic, with no delay or delay to happen (Figure 4).

The connection between them, due to the high complexity index of doing everything together, instead of the strategy of making one flowchart for all products, the modules will be providing according to the use of the application. It just updates what is left in stock to start another day of production and the app starts to interact with the simulator to extract part of the simulation complexity. The mobile application assimilates the best for the stock and production and it makes a search to insert these data into the control system for the next day. In the example, 


\section{$3^{\text {rd }}$ International Conference on Research in SCIENCE, ENGINEERING AND TECHNOLOGY}

\section{Oxford - United Kingdom}

to make $10 \mathrm{~kg}$ of biscuits it is necessary $8 \mathrm{~kg}$ of sour starch, 2 litters of milk, 1,792 grams of vegetable fat, 272 grams of salt and 16 eggs, this amount of ingredients is automatically mixed in the mixer machine and taken to the manual multidrop depositor where its maximum capacity is $15 \mathrm{~kg}$. The process started with the condition that all stocks were full, vegetable fat and salt were calculated per gram, sour starch per kilo, milk represented per litter and eggs per unit. The time taken for weigh a mass and the main mixture is approximately 30 minutes. The biscuit modelling process, which is manual, takes about 5 minutes, followed by 26 minutes for baking 8 kilos of biscuits and the cooling process takes a maximum of 5 minutes.

Figure 4: Traditional process Simulation Module

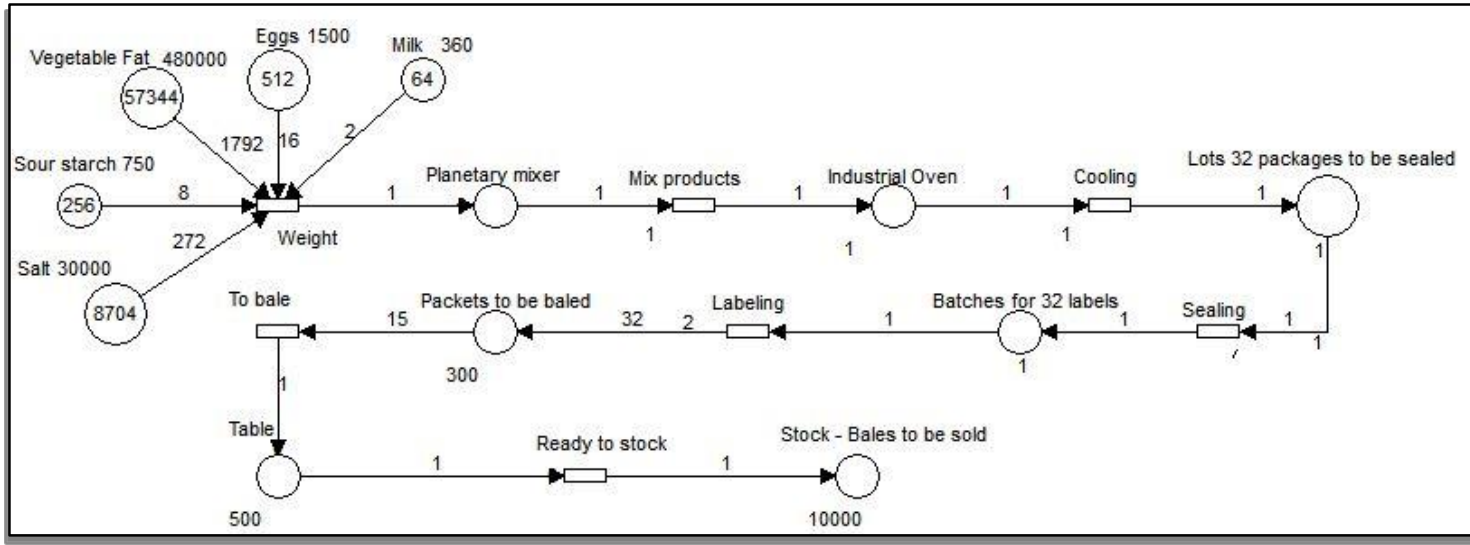

Source: Authors, 2020.

It can be emphasized that weighing, sealing, labelling and baling was calculated in an average time per package. The process is done by volume, in other words, when there is a large quantity of products, those standards can be changed and adapted by the simulator. The manufactured batches are stored in a stock location. The quantity of each bale is 15 units for 200 grams packages and 30 units for 100 grams packages. In the entire production method of Delicate ${ }^{\circledR}$ biscuits, several bottlenecks were found since the beginning of the process to the final stage. Several scenarios of possible changes were thoroughly analysed:

- Scenario I - Current Situation: the scenario presented has a total of 32 batches, 2 days of manufacture, as shown in Figure 4. It can be seen that it was not possible to complete the 68 bales, considering that 4 packages of biscuits remained at the end of the production and can be used for individual sales or wait for the next production of the same flavour to complete the bale. One way to increase production is to purchase a second oven, since it is possible to mix a larger volume of dough in the mixer machine and manual multidrop depositor. It was observed the time for weighing and sealing can be improved, and the ideal scenario would be the purchase of automated machines with automatic processes, which requires a high investment. In relation to product labelling, it was observed that the purchase of custom bags can cut this process off because it would be possible to print the information of the packages in the sealing process. The main problem of this process is the purchasing of the quantity of custom bags for a better price, the same amount should be purchased almost 20 times more, being impracticable for the current size of the company. 


\section{$3^{\text {rd }}$ International Conference on Research in SCIENCE, ENGINEERING AND TECHNOLOGY}

- Scenario II - Custom Bags: The essential improvement would be in the purchase of personalized plastic bags because the production information can be made through the sealer machine. This procedure will eliminate the labelling process. The estimated time earned is on average 1 hour and 30 minutes, in other words, 5 seconds for each package, being analysed in the total 1,024 packages. By acquiring the personalized plastic bags, this time will decrease considerably because it eliminates two processes, stamp and labelling. The estimate after the sealing would be to bale directly. In the current process of the company, there is a delay to organize the packages of biscuits after sealing, conduct the labelling and just after that, it would be possible to carry out the process of baling. In that way, there was a considerable time reduction in labelling, about a $18 \%$ improvement in production speed. The relevance of this result can be enhanced if contemplate that, even being an average of 5 seconds for manual labelling, this time could be longer, since there are several factors for this, such as: rest break, distractions, manufacturing errors and the fatigue for several repetitions.

- Scenario III - Industrial Oven: According to the company's current manufacturing process, some possible improvements were analysed, and the essential is the purchase of a new industrial oven. It was made an analysis using the result obtained by the Petri Net and with this acquisition it is possible to increase production. It was evidenced that, at this moment, it is not interesting to buy another industrial oven in order to increase productivity, because it would be necessary to hire more employees as there would be no significant gains in relation to the speed of weighing productivity. But, on the other hand, it is really interesting to note, as there is only one oven, in case of possible stops, it would be interesting to buy another one, as a second option, so there would be no stop in the production process.

\subsection{Optimization Proposal}

Due to the numbers obtained in the simulation, the resource optimization proposal is mainly aimed on the acquisition of personalized plastic bags, which is the biggest bottleneck found in the company, as it is a completely manual process performed by only two employees. By implementing this process in the company, there would be an improvement, which is essential in a company like this, a food factory that values quality, a fast and agile delivery to the sellers and clients due to the short shelf life products. The price of custom plastic bags varies according to the quantity ranging between $\mathrm{R} \$ 371,79$ to $\mathrm{R} \$ 256,96$ (Brazilian Real - BRL) according to the budget of the bag's supplier $25 \times 35$ on 16 March of 2020. Another recommendation of true value is the purchase of a new industrial oven in consideration of that can prevent a complete stoppage of production in case of preventive and/or corrective maintenance. A solution estimated around R\$20,000.00 (BRL). However, it can be crucial for the business. The graphical analysis in Figure 5 illustrates the main gap of the process, the number of packages to be baled during the Delicate ${ }^{\circledR}$ process production for 2 days. 


\section{$3^{\text {rd }}$ International Conference on Research in SCIENCE, ENGINEERING AND TECHNOLOGY}

Figure 5: Packages to be baled graphical analysis

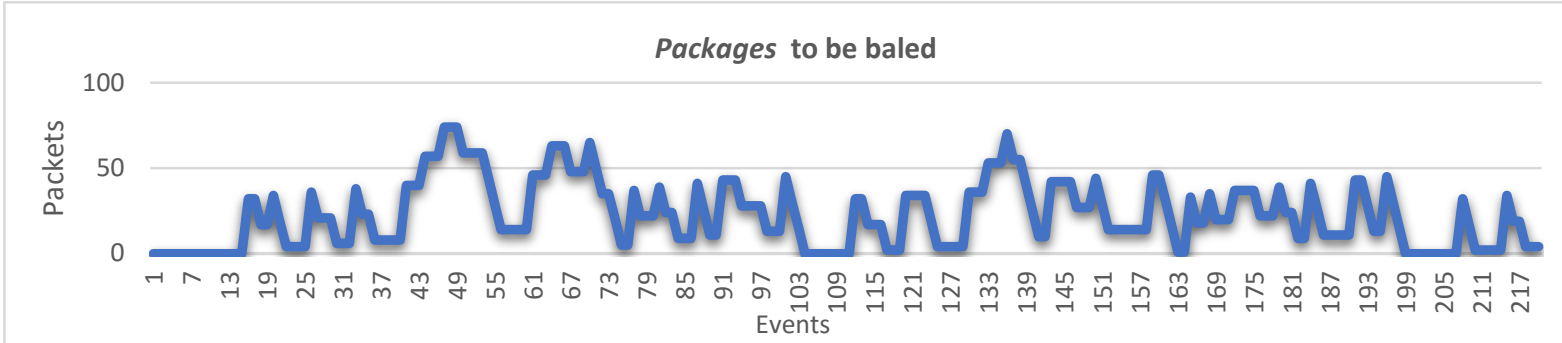

Source: Authors, 2020.

The Delicate ${ }^{\circledR}$ spent more time to do this procedure because one step before, in labelling process, the company needs one employee dedicated to make this action, which is a wasting of time if the packages were customized and could be printed on the sealer. Overall, the employee spent more time on labelling the packages of biscuits than baling the bags in the given period. If consider that each package spent 5 seconds to be labelled, in the presented example with 1,024 produced in these days, which could save at least 85 minutes in this task, the percentage of wasted work time is $9 \%$. In terms of productivity, weekly remain relatively stable about $22.5 \%$. From this point, the proportion of wasted hours was at about 4 hours.

In terms of fluctuation, the rate was not remained relatively stable in the period of this measure. From this point, the proportion of packages climbed gradually to the peak at about 74 to be baled (at point 49) and after which it dropped to zero (at point 106). A similar growth in the other period was evident reaching 70 packages (at point 136) and to 1 package (at point 164), a very large oscillation. Therefore, it becomes necessary to purchase personalized plastic bags to eliminate this process and obtain a huge gain of productivity since the employee could dedicate his time in other activities in the period given.

\section{Conclusion}

The main objective of this research was to improve the procedures and build a new sales process to enable achieve new customers. The company has a large-scale for massive growth and expansion through process improvement and future machinery purchases. Nowadays, the company operates with simple machinery and not too much technology, which slows down the company's growth and development. Most products require manual works throughout the production cycle, which delays very much the final process.

In this research, the proposed model answers a set of three questions. Firstly, the proposed sales process enables information by making data available to all employees, administrators, internal and external staffs. Many times, the departments face a difficulty with lack of knowledge of the customers' history. Then, in the Delicate ${ }^{\circledR}$ production process was identified that with the purchase of personalized plastic bags, to avoid manual labelling of the biscuits, the company will optimize the process by $9 \%$, which may be even better, as the employee today fully dedicated to this role could remain free to work in another function within the company. Finally, it is possible to identify an important detail, the main machinery gap, as the industrial oven is unique and powerful requirement. 


\section{$3^{\text {rd }}$ International Conference on Research in SCIENCE, ENGINEERING AND TECHNOLOGY}

11-13 March, 2021

\section{Oxford - United Kingdom}

Even if there is an inspection and need of prevention of the machinery in any period for any reasons, like corrective maintenance, it would be necessary to interrupt the process of baking biscuits because there is no other machinery to be replaced it. Although it is a substantial investment, and it could be vital to the continuity of the company in the production. With the Petri Net simulation, it was evaluated that it is not interesting to purchase another industrial oven for operation, in parallel with the existing one, unless to increase production. And in that case would be necessary to hire more employees to back up this decision. To increase reliability and validity of the outcome data, more time and interviews with users, the company employees would absolutely benefit the evaluations and might lead to more fine-tuned proposals.

\section{References}

[1] UNCTAD (2016). Science, Technology and Innovation Policy Review of the Islamic Republic of Iran. Available at http://unctad.org/en/PublicationsLibrary/dtlstict20163_en.pdf. Accessed 21 March 2020.

[2] International Finance corporation - IFC (2015). IFC and Small and Medium Enterprises. Available at: https://www.ifc.org/wps. Accessed on 17 March 2020.

[3] Cassandras, C. G. and Lafortune, S. (2009) Introduction to Discrete Event Systems. 2. ed. New York: Springer.

[4] Lisboa, A. C., De Souza, F. H. B., Ribeiro, C. M., Maia, C. A., Saldanha, R. R., Castro, F. L. B. and Vieira, D. A. G. (2019) On Modelling and Simulating Open Pit Mine Through Stochastic Timed Petri Nets. IEEE Access, v. 7, p. 112821 - 112835.

[5] Reis, T., Daryane, J. and Souza, F. H. B. (2018) Discrete Events Systems For Controlling The Production Process in a Dairy Industry. Proceedings in: XXXVIII Encontro Nacional de Engenharia de Produção. Maceió, 2018. 